\title{
Cohesion of $\gamma$-quinacridone and 2,9-dimethylquinacridone in the solid state
}

\author{
Takatoshi Senju, Masataka Sakai, Jin Mizuguchi* \\ Department of Applied Physics, Graduate School of Engineering
}

Yokohama National University

79-5 Tokiwadai, Hodogaya-ku, 240-8501 Yokohama, Japan

\begin{abstract}
Quinacridones are industrially important red pigments characterized by $\mathrm{NH}^{\cdots} \mathrm{O}$ intermolecular hydrogen bonds. Among these, unsubstituted $\gamma$-quinacridone $(\gamma$-QA) and 2,9-dimethylquinacridone (2,9-DMQA) are the major products used widely in painting and imaging industries. Our recent structure analysis revealed that the $\mathrm{NH} \cdots \mathrm{O}$ hydrogen bond in 2,9-DMQA is slightly weaker as compared with that of $\gamma$-QA, although the former is generally known to be more stable against heat and light irradiation than the latter. In order to clarify this point, the cohesion of $\gamma$-QA and 2,9-DMQA has been investigated on the basis of the energy partition analysis of semi-empirical molecular orbital calculations, using the X-ray $x, y$ and $z$ coordinate sets. The $\mathrm{NH}^{\cdots} \mathrm{O}$ hydrogen bond is found to be the major intermolecular force in $\gamma$-QA and 2,9-DMQA, as expected.
\end{abstract}


However, in 2,9-DMQA, an additional interaction is also recognized due to diagonal pairs along the stacking axis. Then, it follows that the sum of the hydrogen bond and the diagonal-pair interactions in 2,9-DMQA exceeds the total cohesive force of $\gamma$-QA, showing a higher stabilization of the former than the latter.

Keywords: Quinacridone; Energy partition; Semi-empirical molecular orbital calculations; Hydrogen-bonded pigments; Cohesive force

* Corresponding author. Tel./Fax: +81 453393369.

E-mail address: mizu-j@ynu.ac.jp (J. Mizuguchi) 


\section{Introduction}

Quinacridones (QAs) are industrially important red pigments used widely in painting and imaging industries [1]. QAs are characterized by $\mathrm{NH} \cdots \mathrm{O}$ intermolecular hydrogen bonds as found in indigo and diketopyrrolopyrroles [1]. The QA molecule is small and thus colored only pale yellow in solution. Nevertheless, QA exhibits a vivid red color in the solid state. Therefore, it has often been pointed out that the $\mathrm{NH} \cdots \mathrm{O}$ intermolecular hydrogen bond plays a crucial role in the color generation in the solid state and also that the hydrogen bond imparts a polymer-like stability to QA. However, details on the role of hydrogen bonds for the color generation remained unclarified. We have tackled this problem on the basis of the crystal structure of the following QAs and interpreted its origin in terms of the excitonic interactions between transition dipoles [2]. We have namely studied three kinds of QA compounds with different hydrogen bond forming characteristics: unsubstituted $\gamma$-quinacridone ( $\gamma$-QA: pigment violet 19$)$ with two NH groups, mono- $\mathrm{N}$-methylquinacridone (MMQA) with one $\mathrm{NH}$ and one $\mathrm{CH}_{3}$, and $N, N^{\prime}$-dimethyl-quinacridone (DMQA) with two $\mathrm{CH}_{3}$ groups. The number of the $\mathrm{NH} \cdots \mathrm{O}$ intermolecular hydrogen bond per molecule is four, two and zero for $\gamma$-QA, MMQA and DMQA, respectively.

Then, we encountered another problem associated with the stability issue of $\gamma$-QA and 
2,9-dimethylquinacridone (2,9-DMQA: pigment red 122) where the $\mathrm{NH} \cdots \mathrm{O}$ hydrogen bond plays an important role in the cohesion in the solid state. The light and heat stability of 2,9-DMQA is generally known to be better than that of $\gamma$-QA. This happens although the $\mathrm{NH} \cdots \mathrm{O}$ hydrogen bond (i.e. the strongest intermolecular interaction in the solid state) is stronger in $\gamma$-QA than in 2,9-DMQA as described below. For this reason, an attempt was made in the present investigation to discuss the cohesion in $\gamma$-QA and 2,9-DMQA in terms of energy partition analysis of semi-empirical molecular orbital calculations on the basis of the crystal structure.

The structure of $\gamma$-QA has variously been studied by Potts et al. [3] and also by us [4]. On the other hand, the structure of 2,9-DMQA has recently been reported by us [5]. The former structure is characterized by three-dimensional, $\mathrm{NH} \cdots \mathrm{O}$ hydrogen bonds in a fashion "hunter's fence"; whereas the two-dimensional network is the case in the latter one. The N/O distance of the hydrogen bond is $2.721 \AA$ in $\gamma$-QA while $2.849 \AA$ in 2,9-DMQA. Furthermore, the NH/O angles in $\gamma$-QA and 2,9-DMQA are 163 and $150^{\circ}$, respectively. The short $\mathrm{N} / \mathrm{O}$ distance and the close angle to $180^{\circ}$ are often a good measure of the strength of the hydrogen bond [6]. As judged from the above geometry, the hydrogen bond of $\gamma$-QA is clearly stronger than that of 2,9-DMQA. 


\section{Energy Partition Analysis for Intermolecular Interactions}

The intermolecular interactions can macroscopically be characterized by sublimation or melting point, hardness of the material as well as solubility in solvents. In our attempt to obtain microscopic information on the intermolecular interactions, we noticed that the two-center integral involved in semi-empirical molecular orbital (MO) calculations could be a measure of intermolecular interactions if the crystal structure is known in advance. We have variously examined the validity of the present method for dithioketopyrrolopyrroles [7], thioindigos [8], tetrathiobenzoquinones [9] and magnesiumphthalocyanine [10].

In semi-empirical MO calculations, the differential overlap is neglected, so that the total energy of a molecule $\left(E_{\text {total }}\right)$ can be partitioned into one-center integral $\left(E_{i}\right)$ of the $i$ th atom and two-center integral $\left(E_{i j}\right)$ of the bonded $i$ th and $j$ th atoms as shown in Eq. (1) $[11]$.

$$
E_{\text {total }}=\sum_{i} E_{i}+\sum_{i<j} E_{i j}
$$

where the two-center energy is further divided into the resonance energy $\left(E_{\text {res }}\right)$, exchange energy $\left(E_{\text {exc }}\right)$ and Coulomb energy $\left(E_{e l}\right)$.

$$
E_{i j}=E_{r e s}+E_{e x c}+E_{e l}
$$

It was Hirano and Osawa $[11,12]$ who proposed an application of the two-center energy 
for chemical bonding problems. Since the two-center integral concerns the energy between bonded atom-pairs, it is directly correlated with the bond energy, although it is not exactly the same. This means that the energy term due to orbital overlap approximately corresponds to the covalent bond while the electrostatic term is related to the ionic bond.

Based upon the pioneer work of Hirano and Osawa, we believed that the present two-center integral could also provide us with information on intermolecular interactions if we applied it for non-bonded atom-pairs between molecules by regarding a pair of molecules as a supermolecule, for example, as shown in Fig. 2. In the evaluation procedure, we specify a given molecule in the lattice and extract typical molecule-pairs composed of the specified molecule and its nearest-neighbor. For each pair, we carry out the energy partition analysis and then list up all atom-pairs with significant interactions. In this way, we can characterize the intermolecular bonding state. However, it should be remembered that the present method is solely powerful for intermolecular bonds that are covalent or ionic in nature and is obviously powerless for "van der Waals" interactions due to instantaneous dipole moments in a statistical sense.

As for the reliability of the present method, we say that this method is as much reliable as that of $\mathrm{MO}$ calculations for geometry optimization and spectroscopic 
calculations, because the energy partition is just the breakdown of the total electronic energy into one and two-center components. In other words, if one finds the MO calculations meaningful for a given system, one must believe that the energy partition analysis also makes sense with no further assumption. It should additionally be noted that the present method is specific of semi-empirical MO calculations and is intractable with $a b$ initio calculations, because the total energy cannot be partitioned into one center and two-center integrals in ab initio calculations as shown in Eq. 1.

\section{Experiment}

\subsection{Materials}

$\gamma$-QA and 2,9-DMQA were obtained from Ciba Specialty Chemicals and Dainippon Ink and Chemicals, Inc., respectively. The products were purified twice by sublimation, using a two-zone furnace [13].

\subsection{Thermogravimetric analysis}

TGA (Thermogravimetric Analysis) measurements were made on powdered samples under vacuum by means of a Rigaku Thermo Plus 8230 at a heating rate of $10^{\circ} \mathrm{C} / \mathrm{min}$. 


\subsection{Molecular orbital calculations - Programs and Calculation Procedure-}

WinMOPAC Ver. 3.5.1a [14] was used for energy partition analysis. The X-ray coordinates were used for non-H atoms, while geometry was optimized for the $\mathrm{H}$-atoms. The calculations were carried out for molecule-pairs in $\gamma$-QA and 2,9-DMQA by specifying the keywords "1SCF" and "ENPART".

\section{Results and discussion}

\subsection{Thermogravimetric analysis}

Fig. 3 shows the weight loss of $\gamma$-QA and 2,9-DMQA as a function of temperature. QA begins to sublime at about $310{ }^{\circ} \mathrm{C}$ while around $340{ }^{\circ} \mathrm{C}$ in 2,9 -DMQA. The sublimation temperature is higher in 2,9-DMQA than in $\gamma$-QA by about $20-30{ }^{\circ} \mathrm{C}$, suggesting that the cohesion is stronger in 2,9-DMQA than in $\gamma$-QA. However, reverse is the case as regards the strength of the hydrogen bond according to the X-ray structure analysis $[4,5]$. This suggests us to assume that other intermolecular interactions are also involved in 2,9-DMQA besides $\mathrm{NH} \cdots \mathrm{O}$ hydrogen bonds.

\subsection{Crystal structure of $\gamma-Q A$ and 2,9-DMQA and their representative molecule-pairs}

Table 1 details the crystallographic parameters for $\gamma$-QA [4] and 2,9-DMQA [5]. In 
both compounds, there are four $\mathrm{NH} \cdots \mathrm{O}$ intermolecular hydrogen bonds per molecule between the $\mathrm{NH}$ group of one molecule and the $\mathrm{O}$ atom of the neighboring one. The skeleton of both $\gamma$-QA and 2,9-DMQA is entirely planar. However, the molecular arrangement is strikingly different as shown in Fig. 4. The QA molecules are arranged in a fashion "hunter's fence" characterized by a three-dimensional hydrogen bond network where one molecule is hydrogen-bonded to four different molecules (Fig. 4(a)). On the other hand, all molecules of 2,9-DMQA are basically hydrogen-bonded on the molecular plane as characterized by the two-dimensional hydrogen bond network where one molecule is hydrogen-bonded to two neighboring molecules (Fig. 4(b)). The hydrogen-bond parameters are given in Introduction.

In $\gamma$-QA, two kinds of molecule pairs (pairs 1 and 2) are extracted from the crystal lattice as shown in Fig. 5. These are designated as "cross pair" (hydrogen-bonded) and "stack pair". On the other hand, in 2,9-DMQA, three representative pairs (pairs 3, 4 and 5) are shown in Fig. 6: pair 3 (hydrogen-bond pair), pair 4 (stack pair) and pair 5 (diagonal pair).

\subsection{Evaluation of the intermolecular forces}

The results of the energy partition for $\gamma$-QA and 2,9-DMQA are given in Table 2. In 
pair 1 of $\gamma$-QA, there is an $\mathrm{NH}^{\cdots} \mathrm{O}$ hydrogen bond, in which there exists an attractive force of $-1.032 \mathrm{eV}$ in $\mathrm{O}^{1} \cdots \mathrm{H}^{1}$. The present interaction is stronger than that of indigo $(-0.906 \mathrm{eV})[8]$ which forms the same type of hydrogen bond network ("hunter's fence") [1]. Since the value of $-0.906 \mathrm{eV}$ in indigo correspond to the energy of about $8-10 \mathrm{kcal} / \mathrm{mol}$ [8], the hydrogen bond in $\gamma-\mathrm{QA}$ is quite strong. This bond is of the covalent nature rather than of the ionic one, as judged from the ratio $E_{\mathrm{res}} / E_{\mathrm{el}}=1.12$. On the other hand, pair 2 is a stack pair along the $b$-axis. The value of $E_{\text {res }}$ and $E_{\text {exc }}$ is nearly zero while $E_{\mathrm{el}}$ is found to be $c a 1 / 8$ of the hydrogen bond energy.

The bonding situation is different in 2,9-DMQA. Pair 3 is a hydrogen-bonded pair nearly on the molecular plane (although there is a small step of about $0.5 \AA$ between two molecules). There is a total energy of $-0.931 \mathrm{eV}$ between $\mathrm{O}^{2}$ and $\mathrm{H}^{2}$ atoms. The present energy is smaller than that in $\gamma$-QA by $0.101 \mathrm{eV}$. This is expected in view of the geometry of the $\mathrm{NH} \cdots \mathrm{O}$ hydrogen bond as described in Introduction. The energy breakdown is $E_{\mathrm{res}} / E_{\mathrm{el}}=0.51$, indicating more ionic character than covalent one. In pair 4 (i.e. stack pair), both attractive and repulsive interactions are recognized. Then, it is remarkable to note that there is a strong attractive interaction of $-0.488 \mathrm{eV}$ in pair 5 (i.e. diagonal pair) in which the center-to-center distance amounts to $2.84 \AA$. This is the next strongest energy to the hydrogen bond one in 2,9-DMQA. 
Judging from the energy partition analysis, $\gamma$-QA is stronger in hydrogen bond than 2,9-DMQA and is in good accord with the results of the structure analysis [4,5]. However, in 2,9-DMQA, an additional interaction is also recognized due to the diagonal pairs along the stacking axis. In total, the cohesion is found to be stronger in 2,9-DMQA than in $\gamma$-QA.

\section{Conclusions}

The difference in cohesion between $\gamma$-QA and 2,9-DMQA has been studied by thermogravimetric analysis as well as the energy partition one based upon MO calculations.

1. Sublimation temperature is found to be higher in 2,9-DMQA than in $\gamma$-QA by about 20-30 ${ }^{\circ} \mathrm{C}$, indicating that the cohesion of the former is higher than that of the latter.

2. As judged from the hydrogen bond parameters based upon the crystal structure, the hydrogen bond is stronger in $\gamma$-QA than 2,9-DMQA. This is also supported by the energy partition analysis. However, in 2,9-DMQA, a strong electrostatic attraction force is also operative due diagonal pairs along the stacking axis. The sum of the hydrogen bond and diagonal-pair interactions in 2,9-DMQA is found to exceed the total cohesive force of $\gamma$-QA. This explains why 2,9-DMQA is more stable than QA. 


\section{References}

[1] Herbst W, Hunger K. Industrial Organic Pigments, 2nd ed. Weinheim: VCH; 1997.

[2] Mizuguchi J, Senju T. Submitted to J Phys Chem. A.

[3] Potts GD, Jones W, Bullock JF, Andrews SJ and Maginn SJ. J Chem Soc, Chem Commun 1994;2565-2566.

[4] Mizuguchi J, Sasaki T, Tojo K. Z Kristallogr NCS 2002;217(2):249-250.

[5] Mizuguchi J, Senju T, Sakai M. Z Kristallogr NCS 2002;217(4):525-526.

[6] Pauling L. The nature of the chemical bond and the structure of molecules and crystals: An introduction to modern structural chemistry. 3rd. ed. Ithaca: Cornell University Press; 1962.

[7] Mizuguchi J. Electrophotography (Denshi Shashin Gakkaishi) 1998;37(1):67-73.

[8] Mochizuki M, Senju T, Mizuguchi J. J Imag Soc Jpn 2001;39(4):421-427.

[9] Matsumoto S, Mizuguchi J. Bull Chem Soc Jpn 2001;74(3):471-477.

[10] Mizuguchi J. J Phys Chem A 2001;105(47):10719-10722.

[11] Hirano T, Osawa E. Croatica Chem Acta 1985;57(6):1633-1642.

[12] Koinuma H, Funabashi M, Kishino K, Kawasaki M, Hirano T, Fueki K. Jpn J Appl Phys 1986;25(12):1811-1814.

[13] Mizuguchi J. Krist Tech 1981;16(6):695-700. 
[14] WinMOPAC Version 3.5.1a Kanagawa, Japan: Fujitsu, Ltd. 


\section{Figure captions:}

Fig. 1. Molecular structure of QA (PV19) and 2,9-DMQA (PR122). The superscript numbers on $\mathrm{H}, \mathrm{C}$ and $\mathrm{O}$ atoms are the numbering used for the energy partition analysis.

Fig. 2. Schematic representation of the energy partition analysis. The supermolecule is composed of a pair of formaldehyde molecules. $E_{i j}$ denotes the two center energy for non-bonded $\mathrm{H} / \mathrm{O}$ pairs.

Fig. 3. Weight loss of QA and 2,9-DMQA as a function of temperature.

Fig. 4. Crystal structure: (a) QA and (b) 2,9-DMQA. The dotted lines designate intermolecular $\mathrm{NH}^{\cdots} \mathrm{O}$ hydrogen bonds.

Fig. 5. Two representative molecule-pairs for QA extracted from the crystal lattice: pair 1 (cross pair) and pair 2 (stack pair).

Fig. 6. Three representative molecule-pairs for 2,9-DMQA extracted from the crystal lattice: pair 3 (hydrogen-bonded pair), pair 4 (stack pair) and pair 5 (diagonal pair). 
Table 1

Crystallographic parameters

\begin{tabular}{lll}
\hline & $\mathrm{QA}^{\mathrm{a}}$ & $2,9-\mathrm{DMQA}^{\mathrm{b}}$ \\
\hline Formula & $\mathrm{C}_{20} \mathrm{H}_{12} \mathrm{~N}_{2} \mathrm{O}_{2}$ & $\mathrm{C}_{22} \mathrm{H}_{16} \mathrm{~N}_{2} \mathrm{O}_{2}$ \\
Crystal system & monoclinic & triclinic \\
Space group & $P 2_{1} / c$ & $P-1$ \\
$Z$ & 2 & 1 \\
Molecular weight & 312.32 & 340.37 \\
$a(\AA)$ & $13.70(1)$ & $3.865(3)$ \\
$b(\AA)$ & $3.84(1)$ & $6.372(3)$ \\
$c(\AA)$ & $13.35(2)$ & $15.78(2)$ \\
$\alpha\left({ }^{\circ}\right)$ & 90 & $93.94(6)$ \\
$\beta\left({ }^{\circ}\right)$ & $100.09(9)$ & $91.51(8)$ \\
$\gamma\left({ }^{\circ}\right)$ & 90 & $100.00(6)$ \\
$V\left(\AA^{3}\right)$ & 691.5 & 381.5 \\
$T(\mathrm{~K})$ & 223 & 123 \\
$R_{1}$ & 0.073 & 0.056 \\
\hline
\end{tabular}

${ }^{\mathrm{a}}$ Ref. $4 .{ }^{\mathrm{b}}$ Ref. 5.

Table 2

Energy partition analysis. The atom numbering is shown in Fig. 1.

\begin{tabular}{clcccc}
\hline $\begin{array}{l}\text { molecule } \\
\text { pair }\end{array}$ & $\begin{array}{l}\text { atom } \\
\text { pair }\end{array}$ & $E_{\text {res }}(\mathrm{eV})$ & $E_{\text {exc }}(\mathrm{eV})$ & $E_{\text {el }}(\mathrm{eV})$ & $E_{\text {total }}(\mathrm{eV})$ \\
\hline QA & & & & & \\
pair 1 & $\mathrm{H}^{1} / \mathrm{O}^{1}$ & -0.5075 & -0.0724 & -0.4524 & -1.0323 \\
pair 2 & $\mathrm{C}^{1} / \mathrm{C}^{2}$ & -0.0063 & -0.0005 & -0.1390 & -0.1458 \\
2,9-DMQA & & & & & \\
pair 3 & $\mathrm{O}^{2} / \mathrm{H}^{2}$ & -0.3030 & -0.0381 & -0.5901 & -0.9312 \\
pair 4 & $\mathrm{O}^{2} / \mathrm{C}^{3}$ & 0.0001 & 0.0000 & -0.3688 & -0.3687 \\
pair 5 & $\mathrm{O}^{2} / \mathrm{H}^{2}$ & -0.0220 & -0.0023 & -0.4641 & -0.4884 \\
\hline
\end{tabular}


<smiles></smiles>

\section{QA (PV19)}<smiles></smiles>

\section{2,9-DMQA (PR122)}

Fig. 1. Molecular structure of QA (PV19) and 2,9-DMQA (PR122). The superscript numbers on $\mathrm{H}, \mathrm{C}$ and $\mathrm{O}$ atoms are the numbering used for the energy partition analysis.

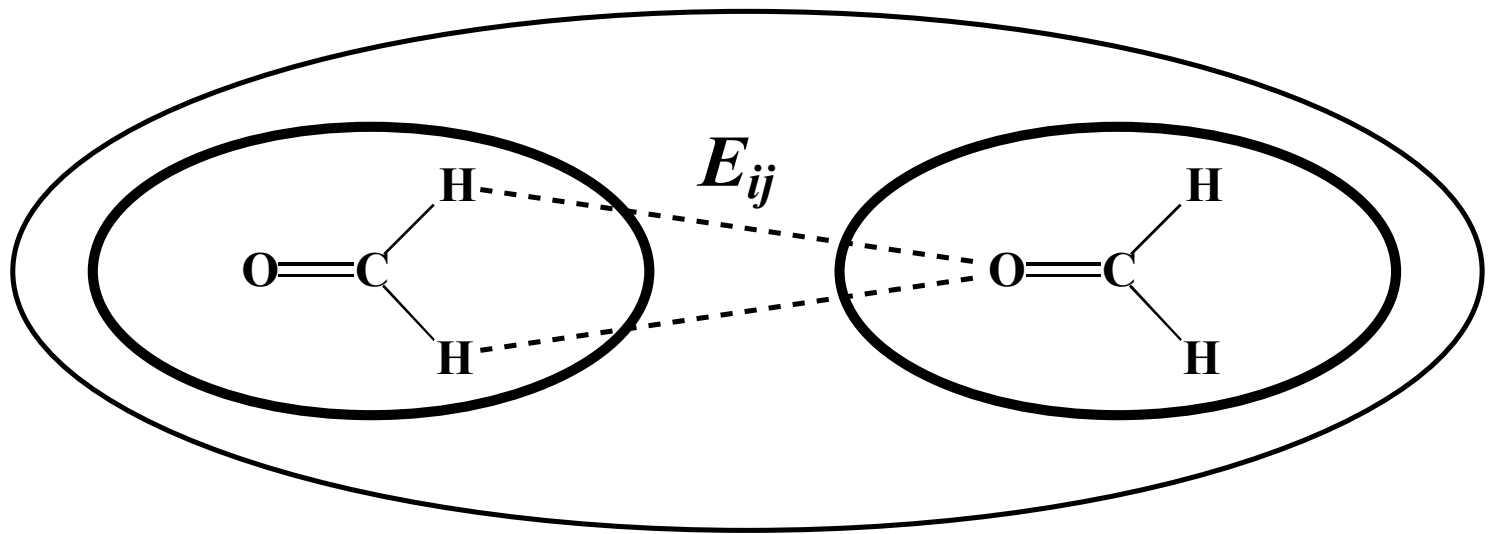

Fig. 2. Schematic representation of the energy partition analysis. The supermolecule is composed of a pair of formaldehyde molecules. $E_{i j}$ denotes the two center energy for non-bonded $\mathrm{H} / \mathrm{O}$ pairs. 


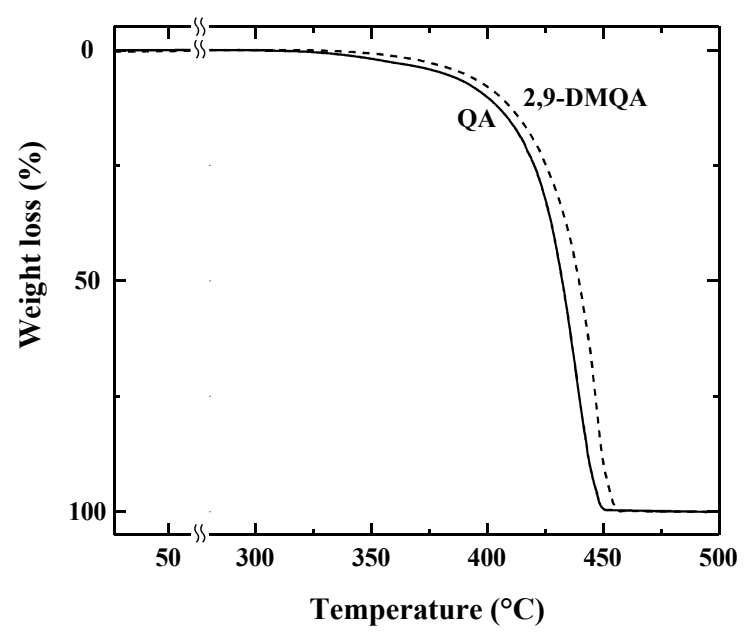

Fig. 3. Weight loss of QA and 2,9-DMQA as a function of temperature.

(a)

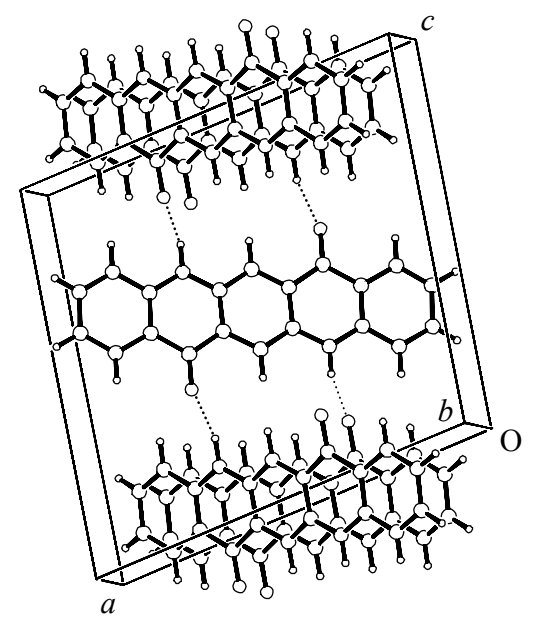

(b)

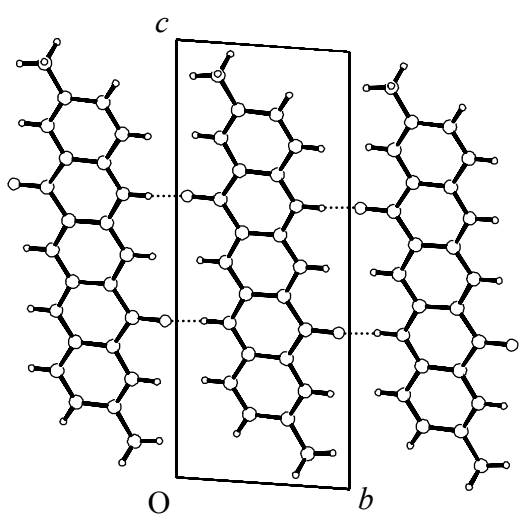

Fig. 4. Crystal structure: (a) QA and (b) 2,9-DMQA. The dotted lines designate intermolecular $\mathrm{NH}^{\cdots} \mathrm{O}$ hydrogen bonds. 


\section{Top view}

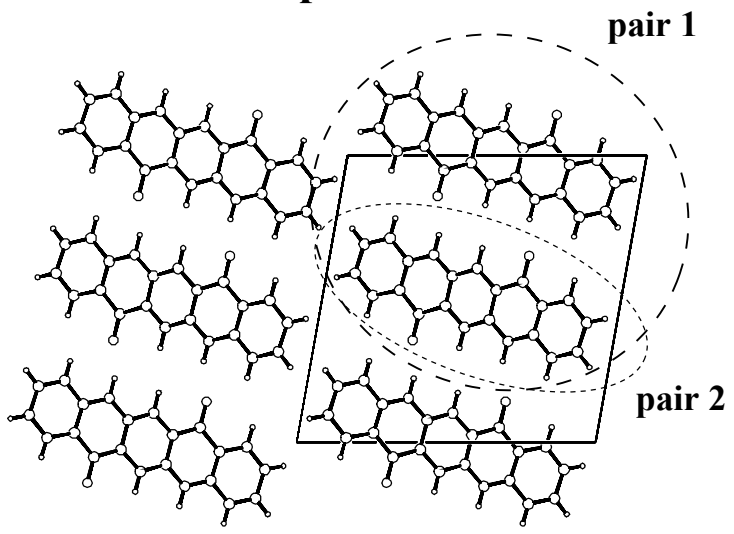

Side view

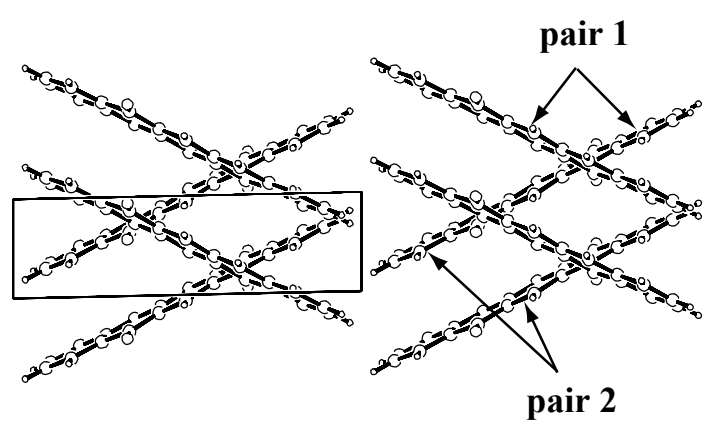

Fig. 5. Two representative molecule-pairs for QA extracted from the crystal lattice: pair 1 (cross pair) and pair 2 (stack pair).

\section{Top view}

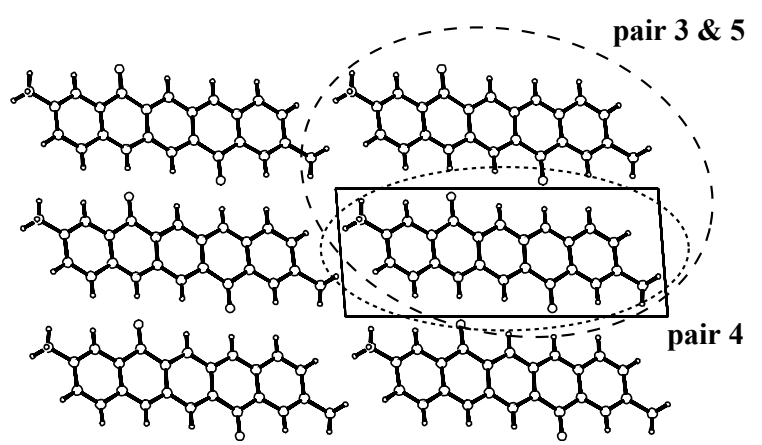

Side view

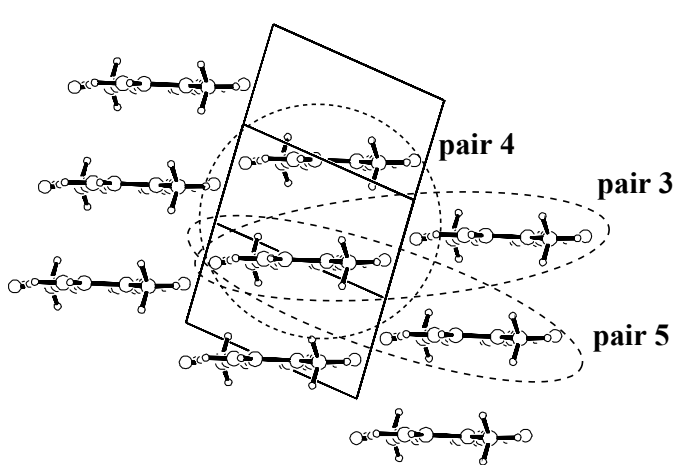

Fig. 6. Three representative molecule-pairs for 2,9-DMQA extracted from the crystal lattice: pair 3 (hydrogen-bonded pair), pair 4 (stack pair) and pair 5 (diagonal pair). 